\section{Tandem Concurrent Processes: One-Pot Single- Catalyst Biohydrogen Transfer for the Simultaneous Preparation of Enantiopure Secondary Alcohols}

Fabricio R. Bisogno, ${ }^{\dagger}$ Iván Lavandera ${ }^{\dagger}$ Wolfgang Kroutil ${ }^{\ddagger}$ and Vicente Gotor* ${ }^{*}$

${ }^{\dagger}$ Departamento de Química Orgánica e Inorgánica, Instituto Universitario de Biotecnología de Asturias, University of Oviedo, 33006 Oviedo, Spain; ${ }^{*}$ Department of Chemistry, Organic and Bioorganic Chemistry, University of Graz, Heinrichstrasse 28, 8010 Graz, Austria

\section{E-mail:vggs@fq.uniovi.es}

RECEIVED DATE (will be automatically inserted after manuscript is accepted).

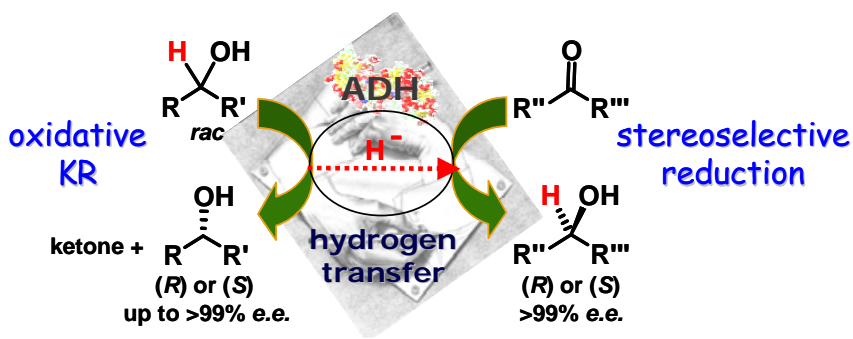

A novel one-pot tandem biohydrogen transfer process to concurrently obtain two enantiopure sec-alcohols is presented; thus, using a suitable single enzyme and a catalytic amount of cofactor several interesting building blocks could be easily achieved in an enantiocomplementary fashion minimizing dramatically the quantity of reagents usually employed in the 'coupledsubstrate' approach.

Several (bio)catalytic methods to synthesize enantiopure secondary alcohols have been developed in the last few years to fulfill the increasing demand of this type of highly valuable compounds. ${ }^{1}$ Among all methodologies described, stereoselective reduction of ketones ${ }^{2}$ and enantioselective oxidation of racemic sec-alcohols ${ }^{3}$ using hydrogen transfer (HT) protocols have extensively been studied due to the mild and simple conditions employed in these transformations. In this context, biocatalyzed HT (also called 'coupled-substrate' approach) employing alcohol dehydrogenases (ADHs) has recently gained increasing relevance. ${ }^{4}$ In these processes, a single enzyme reduces/oxidizes the target substrate sacrificing a small molecule (cosubstrate) like 2-propanol/acetone as hydride donor/acceptor, used in a huge molar excess (at least 10 equiv. compared to 1 equiv. substrate to afford conversions higher than 90\%) due to the reversible character of the reaction.
Scheme 1 Tandem ADH-catalyzed hydrogen transfer concept.

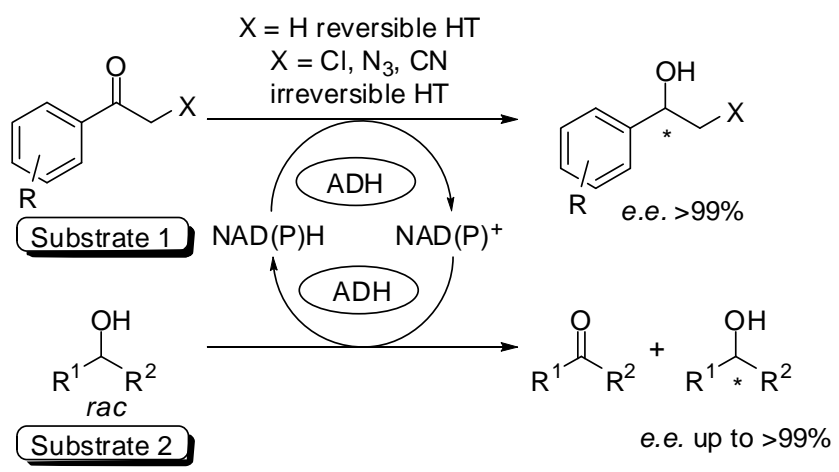

Very recently, it has been described that small activated ketones such as methyl acetoacetate ${ }^{5}$ or chloroacetone ${ }^{6}$ can be employed as cosubstrates in $\mathrm{ADH}$-catalyzed oxidation of alcohols in near stoichiometric amount to achieve complete conversion. Herein, we present a system in which the sacrificial reaction has been turned into a highly valuable transformation, resulting in a one-pot process combining activated ketones with racemic sec-alcohols in order to concurrently obtain two different optically enriched alcohols catalyzed by a single enzyme maximizing thus the atom efficiency environmental factor $E^{7}$ of the process, since no additional reagent is discarded. Therefore, starting from a prochiral ketone and a racemic alcohol, we can obtain two optically pure alcohols (Scheme 1). Another advantage of this system is that the stereoselectivity can be tuned by simply changing the biocatalyst employed.

In a first set of experiments, we studied the influence of the ketone structure on some selected $\mathrm{ADH}$-catalyzed reductions using a low excess of the cosubstrate (2 equiv. of 2-propanol, see Supporting Information). Thus, several ketones were reduced using a Prelog (ADH-A from Rhodococcus ruber $),{ }^{8}$ or an anti-Prelog [ADH from Lactobacillus brevis (LB-ADH) $]^{9}$ enzyme. It could be observed that non-activated ketones like acetophenone afforded 50\% conversion. When $p$-substituted acetophenones were reduced, electron donating groups provided low conversions $(<30 \%)$, while electron withdrawing substituents afforded conversions about $80 \%$. Moreover, ketones with an electron withdrawing group at $\alpha$-position such as $\alpha$-chloroacetophenone furnished quantitative conversions. These results can be explained due to the different oxidationreduction potentials $\left(\Delta E^{O}\right)$ between the ketone/alcohol pair with regards to the 2-propanol/acetone counterpart. ${ }^{10}$ It has been shown that $\alpha$-halohydrins are stabilized via intramolecular $\mathrm{H}$-bond between the alcohol moiety and the halogen atom, ${ }^{11}$ therefore preventing the $\mathrm{ADH}$-catalyzed oxidation. $^{6}$ 
Table 1. Tandem Concurrent Biohydrogen Transfer Using Activated Ketones and 2-Octanol ${ }^{a}$

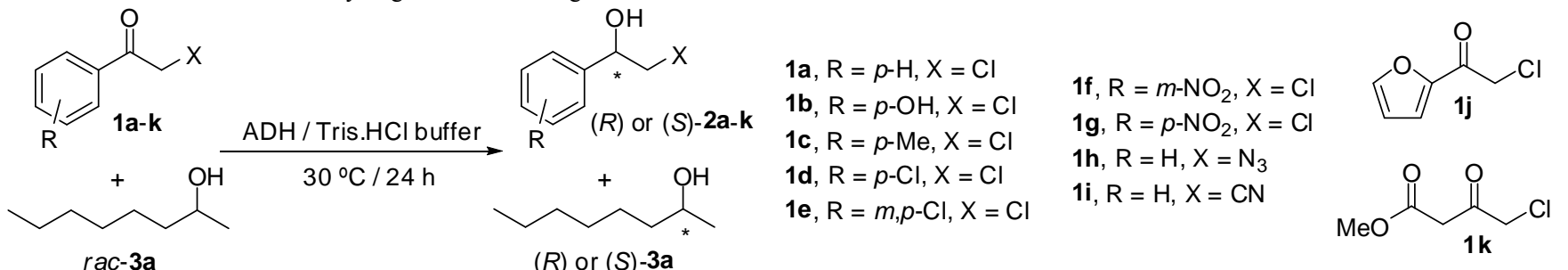

\begin{tabular}{|c|c|c|c|c|c|c|c|c|c|}
\hline \multirow{3}{*}{ entry } & \multirow{3}{*}{ ketone } & \multicolumn{4}{|c|}{ Prelog ADH } & \multicolumn{4}{|c|}{ anti-Prelog ADH } \\
\hline & & \multirow[t]{2}{*}{ enzyme } & \multicolumn{2}{|l|}{ 2a-k } & \multirow{2}{*}{$\begin{array}{l}\text { 3a } \\
e e(\%)^{c}\end{array}$} & \multirow[t]{2}{*}{ enzyme } & \multicolumn{2}{|l|}{$2 a-k$} & \multirow{2}{*}{$\begin{array}{l}3 \mathbf{a} \\
e e(\%)^{c}\end{array}$} \\
\hline & & & conv $^{b}$ & $e e(\%)^{c}$ & & & conv $^{b}$ & $e e(\%)^{c, d}$ & \\
\hline 1 & 1a & ADH-A & 81 & $>99(R)^{d}$ & $83(R)$ & LB-ADH & 90 & $>99(S)$ & $98(S)$ \\
\hline 2 & $1 b^{e}$ & ADH-A & 57 & $>99(R)^{d}$ & $44(R)$ & LB-ADH & 83 & $>99(S)$ & $79(S)$ \\
\hline 3 & 1c & ADH-A & 97 & $>99(R)$ & $94(R)$ & LB-ADH & 94 & $>99(S)$ & $92(S)$ \\
\hline 4 & 1d & ADH-A & 85 & $>99(R)^{d}$ & $88(R)$ & LB-ADH & 86 & $>99(S)$ & $88(S)$ \\
\hline 5 & $1 \mathrm{e}$ & $\mathrm{ADH}-\mathrm{A}^{f}$ & 93 & $99(R)^{d}$ & $98(R)$ & PR2 & 90 & $99(S)$ & $>99(S)$ \\
\hline 6 & $\mathbf{1 f}^{e}$ & ADH-A & 89 & $>99(R)^{d}$ & $99(R)$ & LB-ADH & 90 & $>99(S)$ & $98(S)$ \\
\hline 7 & $1 g$ & ADH-A & 91 & $>99(R)^{d}$ & $>99(R)$ & LB-ADH & 90 & $>99(S)$ & $>99(S)$ \\
\hline 8 & $1 h^{e}$ & ADH-T & 73 & $>99(R)^{d}$ & $70(R)$ & PR2 & 84 & $>99(S)$ & $90(S)$ \\
\hline 9 & $1 \mathbf{i}$ & $\mathrm{ADH}-\mathrm{T}^{e}$ & 78 & $>99(S)$ & $72(R)$ & $g$ & -- & -- & -- \\
\hline 10 & $1 \mathrm{j}$ & $\mathrm{ADH}-\mathrm{A}^{e}$ & 85 & $>99(R)^{d}$ & $94(R)$ & LB-ADH & 87 & $>99(S)$ & $94(S)$ \\
\hline 11 & $1 \mathrm{k}$ & $\mathrm{ADH} \mathrm{T}^{f}$ & $>99$ & $>99(R)^{d}$ & $>99(R)$ & LB-ADH ${ }^{h}$ & $>99$ & $>99(S)$ & $>99(S)$ \\
\hline
\end{tabular}

${ }^{a}$ Enzyme: (3-5 U); [1]: $50 \mathrm{mM}$; [3a]: 90 to $100 \mathrm{mM}$; [NAD(P)H]: 1 mM. ${ }^{b}$ Measured by achiral GC. ${ }^{c}$ Measured by chiral GC or HPLC. ${ }^{d}$ Switch in CahnIngold-Prelog priority (CIP). ${ }^{e}[\mathbf{3 a}]: 45 \mathrm{mM} .{ }^{f}[\mathbf{3 a}]: 180 \mathrm{mM} .{ }^{g}$ Not appropriate ADH found. ${ }^{h}[\mathbf{3 a}]: 400 \mathrm{mM}$.

Taking as an advantage the irreversibility of this HT, we tested the concept in a one-pot tandem protocol to simultaneously obtain two enantiopure sec-alcohols (see Scheme of Table 1). In theory, an irreversible asymmetric reduction is required to achieve a complete kinetic oxidative resolution, thus a molar amount of ketone to be reduced can be equal or slightly higher than the molar amount of alcohol to be oxidized. Thus, mixing an activated ketone (1 equiv.) with a racemic alcohol (1.8-2 equiv.), the selective reduction of the prochiral compound plus the kinetic resolution of the racemate could be achieved via HT by a single enzyme and a catalytic amount of the pyrimidinic cofactor which is internally recycled. Due to the perfect selectivity shown by the biocatalysts utilized, the hydride is abstracted from a single enantiomer of the racemic alcohol, and then exclusively transferred to one stereo-face of the prochiral ketone.

Therefore, several $\alpha$-chloro-, $\alpha$-azido-, and $\alpha$-cyano ketones (1a-k, Table 1) were purchased or synthesized and then combined with racemic 2-octanol (3a). Except for the cyano derivative 1i (entry 9), we were able to find a suitable Prelog and anti-Prelog ADH to obtain enantioenriched or enantiopure $(R)$ - or (S)-3a using activated aliphatic and (hetero)aryl ketones, which were reduced to the corresponding enantiopure alcohols with very high yields. Compounds with electron donating groups in the phenyl ring (1b, entry 2), afforded lower conversions. By simply changing the enzyme, enantiocomplementary products could be achieved. Thus, ADH-A or LB-ADH were usually employed, but in some cases Thermoanaerobacter sp. ADH (ADH-T) ${ }^{12}$ or PR2 provided better results. As an example, we scaled-up the reaction of $\mathbf{2 k}$ with $\mathrm{LB}-\mathrm{ADH}$ up to a substrate concentration of $400 \mathrm{mM}$, showing the great robustness of the system. The obtained $\alpha$-activated alcohols are important precursors of pharmaceutical compounds. For instance, $(S)-\mathbf{2 a}$ is an intermediate for the synthesis of fluoxetine, tomoxetine, and nisoxetine, ${ }^{13}(R)-\mathbf{2 b}$ can be used as precursor of $\beta$-agonists like octopamine or denopamine, ${ }^{14}$ and optically active $\mathbf{2 k}$ is a useful chiral building block for the synthesis of different pharmaceuticals. $^{15}$

On the other hand, several sec-alcohols were resolved using chloro ketone 1e with ADH-A, and 1k with LB-ADH (Table 2). Thus, aromatic (3b-d), aliphatic (3e-f) such as sulcatol, or cycloalkyl (3g) derivatives could be successfully obtained in enantioenriched form via tandem concurrent HT.

Table 2. Resolution of sec-Alcohols via ADH-Catalyzed Tandem HT Mediated by $\alpha$-Chloro Ketones ${ }^{a}$

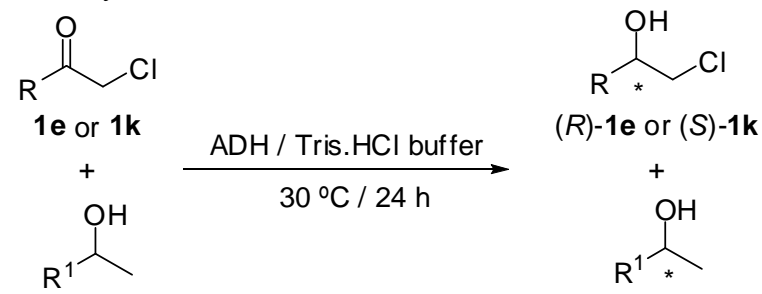

rac-3b-g

$(R)$ or $(S)-\mathbf{3 b}-\mathbf{g}$

3b, $\mathrm{R}^{1}=\mathrm{Ph} ; \mathbf{3 c}, \mathrm{R}^{1}=m-\mathrm{ClC}_{6} \mathrm{H}_{4} ; \mathbf{3 d}, \mathrm{R}^{1}=p-\mathrm{OMeC}_{6} \mathrm{H}_{4}$

3e, $\mathrm{R}^{1}=\mathrm{C}_{9} \mathrm{H}_{20} ; \mathbf{3 f}, \mathrm{R}^{1}=\mathrm{Me}_{2} \mathrm{CH}=\mathrm{CH}\left(\mathrm{CH}_{2}\right)_{2} ; \mathbf{3 g}, \mathrm{R}^{1}=$ cyclohexyl

\begin{tabular}{|c|c|c|c|c|c|c|}
\hline \multirow{3}{*}{ alcohol } & \multicolumn{3}{|c|}{ ADH-A } & \multicolumn{3}{|c|}{ LB-ADH } \\
\hline & \multicolumn{2}{|l|}{$1 \mathrm{e}$} & \multirow{2}{*}{$\begin{array}{l}\text { 3b-g } \\
e e(\%)^{c}\end{array}$} & \multicolumn{2}{|l|}{$1 \mathrm{k}$} & \multirow{2}{*}{$\begin{array}{l}\text { 3b-g } \\
e e(\%)^{c}\end{array}$} \\
\hline & conv $^{b}$ & $e e(\%)^{c, d}$ & & conv $^{b}$ & $e e(\%)^{c, d}$ & \\
\hline $3 \mathbf{b}$ & 90 & $99(R)$ & $99(R)$ & $>99$ & $>99(S)$ & $>99(S)$ \\
\hline $3 \mathbf{c}$ & 85 & $99(R)$ & $81(R)$ & 94 & $>99(S)$ & $87(S)$ \\
\hline 3d & 91 & $99(R)$ & $>99(R)$ & 99 & $>99(S)$ & $>99(S)$ \\
\hline $3 \mathbf{e}$ & 93 & $99(R)$ & $95(R)$ & 97 & $>99(S)$ & $96(S)$ \\
\hline 3f & 95 & $99(R)$ & $>99(R)$ & $>98$ & $>99(S)$ & $>99(S)$ \\
\hline $3 g$ & 94 & $99(R)$ & $98(R)$ & $>98$ & $>99(S)$ & $>99(S)$ \\
\hline
\end{tabular}
mM. ${ }^{b}$ Measured by achiral GC. ${ }^{c}$ Measured by chiral GC. ${ }^{d}$ Switch in CIP.

In summary, we have demonstrated a novel one-pot tandem system to simultaneously obtain two enantiopure sec-alcohols that possesses several advantages: conversion can be easily 
controlled by the amount of racemic alcohol added, a single biocatalyst and catalytic amount of cofactor are used, the selectivity can be tuned by choosing the appropriate enzyme, and the process can be scaled-up. ${ }^{16}$ This is an elegant example that shows how biocatalysis can be applied for the "clean" synthesis of valuable enantiopure compounds maximizing the atom efficiency. ${ }^{7}$

\section{Experimental Section}

General. Alcohol dehydrogenases, ketones 1a, 1d, 1e, 1i, $\mathbf{1 k}$, racemic alcohols 3a, 3b, 3c, 3d, 3e, 3f and 3g, and their corresponding ketones were purchased from commercial sources. $\alpha$-Chloro ketones $\mathbf{1 b}, \mathbf{1 c}, \mathbf{1 f}, \mathbf{1 g}$, and $\mathbf{1 j}$ were synthesized following modified protocols described in the literature. ${ }^{17} \alpha$-Azido ketone $\mathbf{1 h}$ was obtained as published before. $^{8 \mathrm{a}}$ Racemic alcohols $\mathbf{2 a - k}$ were synthesised by conventional reduction from the corresponding ketones $\left(\mathrm{NaBH}_{4}, \mathrm{MeOH}\right.$, room temperature). All other reagents and solvents were of the highest quality available. 1 unit (U) of ADH reduces $1.0 \mu \mathrm{M}$ of acetophenone to 1-phenylethanol per minute at $\mathrm{pH} 7.5$ and $30^{\circ} \mathrm{C}$ in the presence of $\mathrm{NAD}(\mathrm{P}) \mathrm{H}$. Flash chromatography was performed using silica gel 60 (230-400 mesh).

General procedure for the tandem concurrent biohydrogen transfer using activated ketones and 2octanol. In a $1.5 \mathrm{~mL}$ Eppendorf vial, 3-5 U of commercially available $\mathrm{ADH}$ (Lactobacillus brevis $\mathrm{ADH}$, Rhodococcus ruber ADH-A, Thermoanaerobacter sp. ADH or PR2 ADH) in Tris- $\mathrm{HCl}$ buffer $[50 \mathrm{mM}, \mathrm{pH} 7.5,1 \mathrm{mM}$ NAD(P)H, $1 \mathrm{mM}$ $\mathrm{MgCl}_{2}$ for LB-ADH] were mixed with both the racemic 2octanol and the prochiral ketone (1a-k) in a 1.8-2:1 molar ratio respectively (e.g., 90-100 $\mathrm{mM}$ racemic 3a and $50 \mathrm{mM}$ ketone) in a final volume of $0.6 \mathrm{~mL}$. The reaction was incubated at 30 ${ }^{\circ} \mathrm{C}$ and orbital rotation $(150 \mathrm{rpm})$ for $24 \mathrm{~h}$. Then, the reaction was stopped by extraction with ethyl acetate $(2 \times 0.6 \mathrm{~mL})$. The organic layer was separated by centrifugation $(2 \mathrm{~min}, 13000$ rpm) and dried $\left(\mathrm{Na}_{2} \mathrm{SO}_{4}\right)$. Conversions and enantiomeric excesses of the corresponding alcohols were determined by GC or HPLC analysis using an achiral or chiral stationary phase, respectively.

General procedure for the resolution of sec-alcohols via ADH-catalyzed tandem HT mediated by $\alpha$-chloro ketones. In a $1.5 \mathrm{~mL}$ Eppendorf vial, 3-5 $\mathrm{U}$ of commercially available ADH (LB-ADH or ADH-A) in Tris- $\mathrm{HCl}$ buffer [50 mM, pH 7.5, $1 \mathrm{mM}$ NAD(P)H, $1 \mathrm{mM} \mathrm{MgCl}$ for LB-ADH] were mixed with both the racemic alcohol (3b-g, 90-100 $\mathrm{mM})$ and the prochiral ketone (1e for ADH-A or $\mathbf{1 k}$ for LB-ADH, $50 \mathrm{mM}$ ) in a final volume of $0.6 \mathrm{~mL}$. The reaction was incubated at 30 ${ }^{\circ} \mathrm{C}$ and orbital rotation $(150 \mathrm{rpm})$ for $24 \mathrm{~h}$. Then, the reaction was stopped by extraction with ethyl acetate $(2 \times 0.6 \mathrm{~mL})$. The organic layer was separated by centrifugation ( $2 \mathrm{~min}, 13000$ rpm) and dried $\left(\mathrm{Na}_{2} \mathrm{SO}_{4}\right)$. Conversions and enantiomeric excesses of the corresponding alcohols were determined by GC or HPLC analysis using an achiral or chiral stationary phase, respectively.

Acknowledgments. F.R.B. is supported by the Programme Alßan, the European Union Program of High Level Scholarships for Latin America (scholarship No. E07D402519AR). I.L. thanks Principado de Asturias for personal funding (Clarín Program). Financial support from the Spanish Ministerio de Ciencia e Innovación (MICINN, Project CTQ2007-61126/PPQ) is gratefully acknowledged.

Supporting Information Available: Experimental procedures and analytics are detailed. This material is free of charge via the Internet at http://pubs.acs.org.

\section{REFERENCES}

(1) Breuer, M.; Ditrich, K.; Habicher, T.; Hauer, B.; Kesseler, M.; Stürmer, R.; Zelinski, T. Angew. Chem. Int. Ed. 2004, 43, 788

(2) Recent reviews: (a) Wu, X.; Xiao, J. Chem. Commun. 2007, 2449. (b) Ikariya, T.; Blacker, A. J. Acc. Chem. Res. 2007, 40, 1300. (c) Gladiali, S.; Alberico, E. Chem. Soc. Rev. 2006, 35, 226. (d) Ikariya, T.; Murata, K.; Noyori, R. Org. Biomol. Chem. 2006, 4, 393.

(3) Recent bibliography: (a) Modern Biooxidation. Enzymes, Reactions and Applications; Schmid, R. D.; Urlacher, V. B. Eds.; Wiley-VCH: Weinheim, 2007. (b) Arends, I. W. C. E. Catalytic oxidations in Green Chemistry and Catalysis; Sheldon, R. A.; Arends, I. W. C. E.; Hanefeld, U. Eds.; Wiley-VCH: Weinheim, 2007. (c) Lenoir, D. Angew. Chem. Int. Ed. 2006, 45, 3206. (d) Modern Oxidation Methods; Bäckvall, J.-E. Ed.; Wiley-VCH: Weinheim, 2004.

(4) Recent bibliography: (a) Buchholz, S.; Gröger, H. In Biocatalysis in the Pharmaceutical and Biotechnology Industry; Patel, R. N. Ed.; CRC Press: Boca Raton, 2007, p. 757. (b) de Wildeman, S. M. A.; Sonke, T.; Schoemaker, H. E.; May, O. Acc. Chem. Res. 2007, 40, 1260. (c) Moore, J. C.; Pollard, D. J.; Kosjek, B.; Devine, P. N. Acc. Chem. Res. 2007, 40, 1412. (d) Goldberg, K.; Schroer, K.; Lütz, S.; Liese, A. Appl. Microbiol. Biotechnol. 2007, 76, 237. (e) Kroutil, W.; Mang, H.; Edegger, K.; Faber, K. Adv. Synth. Catal. 2004, 346 125. (f) Kroutil, W.; Mang, H.; Edegger, K.; Faber, K. Curr. Opin. Chem. Biol. 2004, 8, 120. (g) Nakamura, K.; Matsuda, T. J. Org. Chem. 1998, 63, 8957.

(5) Peschko, C.; Stohrer, J. Enzyme catalyzed oxidation of secondary alcohols. Wacker Chemie AG, Germany, DE 102006009743, A1 20070906, CAN 2007, 147,321414

(6) Lavandera, I.; Kern, A.; Resch, V.; Ferreira-Silva, B.; Glieder, A.; Fabian, W. M. F.; de Wildeman, S.; Kroutil, W. Org. Lett. 2008, 10, 2155.

(7) (a) Sheldon, R. A. Pure Appl. Chem. 2000, 72, 1233; (b) Trost, B. M. Science 1991, 254, 1471.

(8) This $(S)$-selective enzyme has a strong preference for NADH/NAD ${ }^{+}$. See: (a) Edegger, K.; Gruber, C. C.; Poessl, T. M.; Wallner, S. R.; Lavandera, I.; Faber, K.; Niehaus, F.; Eck, J.; Oehrlein, R.; Hafner, A.; Kroutil, W. Chem. Commun. 2006, 2402. (b) Stampfer, W.; Kosjek, B.; Moitzi, C.; Kroutil, W.; Faber, K. Angew. Chem. Int. Ed. 2002, 41, 1014

(9) This $(R)$-selective enzyme shows a preference for NADPH/NADP ${ }^{+}$. See, for instance: Wolberg, M.; Hummel, W.; Wandrey, C.; Müller, M. Angew. Chem. Int. Ed. 2000, 39, 4306.

(10) Eckstein, M. F.; Peters, M.; Lembrecht, J.; Spiess, A. C.; Greiner, L. Adv. Synth. Catal. 2006, 348, 1591.

(11) Goldstein, T.; Snow, M. S.; Howard, B. J. J. Mol. Spectrosc. 2006, $236,1$. (12) Findrik, Z.; Vasić-Racki, D.; Lütz, S.; Daussmann, T.; Wandrey, C. Biotechnol. Lett. 2005, 27, 1087.

(13) Zhu, D.; Mukherjee, C.; Hua, L. Tetrahedron: Asymmetry 2005, 16, 3275. (14) Lee, D.-M.; Lee, J.-C.; Jeong, N.; Lee, K.-I. Tetrahedron: Asymmetry 2007, 18, 2662

(15) Kizaki, N.; Yasohara, Y.; Hasegawa, J.; Wada, M.; Kataoka, M.; Shimizu, S. Appl. Microbiol. Technol. 2001, 55, 590.

(16) The selection of the substrates was based on their different physical properties. Thus, ketones and aliphatic alcohols can be distilled while the employed aromatic alcohols can be separated using flash chromatography. (17) (a) Mei, Y.; Bentley, P. A.; Du, J. Tetrahedron Lett. 2008, 49, 3802. (b) Lee, J. C.; Bae, Y. H.; Chang, S. K. Bull. Korean Chem. Soc. 2003, 24, 407. 